\title{
Expression profile of mRNAs from human pancreatic islet tumors
}

\author{
L Jin ${ }^{1}$, H Wang ${ }^{1}$, T Narita ${ }^{1}$, R Kikuno ${ }^{2}$, O Ohara ${ }^{2,3}$, N Shihara ${ }^{1,4}$, T Nishigori ${ }^{1}$, \\ Y Horikawa ${ }^{1,4}$ and $\mathrm{J}$ Takeda $^{1,4,5}$ \\ ${ }^{1}$ Laboratory of Molecular Genetics, Department of Cell Biology, Institute for Molecular and Cellular Regulation, Gunma University, Maebashi, \\ Gunma 371-8512, Japan \\ ${ }^{2}$ Department of Human Gene Research, Kazusa DNA Research Institute, Kisarazu, Chiba 292-0818, Japan \\ ${ }^{3}$ Laboratory for Immunogenomics, RIKEN Research Center for Allergy and Immunology, RIKEN Yokohama Institute, Yokohama, Kanagawa 230-0045, \\ Japan \\ ${ }^{4}$ Core Research for Evolutional Science and Technology (CREST), Japan Science and Technology Corporation (JST), Kawaguchi, Saitama 332-0012, \\ Japan \\ ${ }^{5}$ Division of Bioregulatory Medicine, Department of Endocrinology, Diabetes and Rheumatology, Gifu University School of Medicine, Gifu, \\ Gifu 500-8705, Japan \\ (Requests for offprints should be addressed to J Takeda, Department of Endocrinology, Diabetes and Rheumatology, Division of Bioregulatory \\ Medicine, Gifu University School of Medicine, 40 Tsukasa-machi, Gifu 500-8705, Japan; Email: jtakeda@cc.gifu-u.ac.jp)
}

\begin{abstract}
In order to understand the tissue specificity of the endocrine pancreas, it is important to clarify the expression profile of mRNAs in various states of the tissue. A total of 9000 non-redundant expressed genes from human pancreatic islets and insulinoma have so far been determined as expressed sequence tags (ESTs) and deposited in public databases. In the present study towards the identification of a complete set of genes expressed in human pancreatic islets, we have determined 3'-ESTs of 21267 clones randomly selected from a cDNA library of human pancreatic islet tumors. Clustering analysis generated 6157 non-redundant sequences comprising 2323 groups and 3834 singletons. Nucleotide and peptide database searches show that 3103 of them represent known human sequences or homologs of genes identified in other species and 58 are new members of structurally related families. The sequences were classified on the basis of the putative protein functions encoded, and were assigned to the respective chromosome by database analysis. The sequences were also compared with the EST databases (dbEST and EPConDB) including ESTs from normal pancreatic islet, insulinoma, and fetal pancreas. Since 3384 genes were newly found to be expressed in human pancreatic islets and 587 of them were unique to the islets, this study has considerably expanded the catalog of genes expressed in the endocrine pancreas. The larger collection of pancreatic islet-related ESTs should provide a better genome source for molecular studies of differentiation, tissue-specific functions, and tumorigenesis of the endocrine pancreas as well as for genetic studies of diabetes mellitus.
\end{abstract}

Journal of Molecular Endocrinology (2003) 31, 519-528

\section{Introduction}

The pancreatic islets that play the central role in the regulation of glucose homeostasis include $\alpha-, \beta-$, $\delta$-, and PP-cells, all of which develop from common progenitor stem cells (Gordon \& Halban 2001). Glucagon and insulin, which are secreted from $\alpha$ cells and $\beta$ cells respectively, counteract to maintain plasma glucose levels within the normal range.
Although its functional properties in the islets have not been established, somatostatin $(\delta$-cell $)$ may negatively regulate both insulin and glucagon secretion through paracrine mechanisms. Diabetic patients exhibit elevated plasma glucose due to absolute or relative deficiency of insulin, together with inappropriately increased glucagon secretion, implicating desensitization of the $\alpha$ cells in hyperglycemia (Gordon \& Halban 2001). While the 
insulin deficiency in chronic hyperglycemia is paramount, the metabolic derangement is a complex multi-hormonal disturbance.

The differentiation and morphogenesis of pancreatic islets has been well characterized in many laboratories. Most of the information on molecules that regulate cell differentiation and growth are found in the studies of a series of transcription factors (Habener \& Stoffers 1998, Edlund 2002). Functional disorders of this network of regulatory functions might well lead to abnormal organogenesis, growth, or hormone synthesis of the endocrine pancreas. To clarify the molecular mechanisms of tissue-specific functions in both normal and disease states, it is important to understand both the molecular architecture of the endocrine pancreas and the gene products which confer on functional islet cells their ability to differentiate and secrete a unique hormone.

Recently, the genome project has identified $\sim 35000$ genes in human chromosomes (International Human Genome Sequencing Consortium 2001), but a significant fraction of them are hypothetical, revealed only by a computer prediction program. Thus, genetic studies rely largely on public sources of expressed sequence tags (EST) generated by single-pass sequencing of the termini of cDNAs (Adams et al. 1991). Sequences obtained by random selection of cDNA clones from the library can generate a statistical picture of the level and complexity of gene expression in a tissue of interest. We previously reported a preliminary trial of sequencing and characterization of 1000 human pancreatic islet cDNAs (Takeda et al. 1993, 1995). While additional normal pancreatic islet ESTs have now been identified and deposited in the public databases (Bernal-Mizrachi et al. 2003, Kaestner et al. 2003), it is still difficult to obtain the required number of human normal islets of good quality without contamination by exocrine pancreatic tissue for library construction. In this context, additional pure sequences from various human insulinomas have been collected (Bernal-Mizrachi et al. 2003, Kaestner et al. 2003). This approach to accumulating tumor sequences provides a significantly larger set of non-redundant islet sequences, since the tumor tissues generally express a subset of mRNAs at high levels which are expressed at very low levels that can barely be detected in normal tissues. Accordingly, other tissue sources with altered levels of mRNAs should also be similarly searched to increase the number of non-redundant genes.

In this study towards the identification of a complete set of genes expressed in pancreatic islets in both normal and disease states, we have significantly expanded the list of non-redundant islet-related ESTs in the public databases, using a cDNA library of pooled human pancreatic islet tumors. Islet cell tumors exhibit great variability in both clinical manifestations and structural cell features, and often secrete not only cell-specific hormones but also other islet-related hormones. Accordingly, because of the shared features of pluripotency and the varied grade of differentiation, islet cell tumors are especially suitable for efficiently covering the wider spectrum of mRNAs involved in differentiation, tumorigenesis, and cell-specific functions of the endocrine pancreas.

\section{Materials and methods}

\section{Single-pass cDNA sequencing}

An oligo dT-primed unidirectional cDNA library with inserts larger than $\sim 400 \mathrm{bp}$, which was constructed using mRNAs from pooled islet cell tumors and the Uni-ZAP XR vector system, was purchased from Stratagene (La Jolla, CA, USA). Although the characteristic features of the tumor tissues used for library construction were not available from the manufacturer, admixture of the different islet tumors should increase the complexity of the cDNAs. Plasmid DNAs were prepared as described previously (Takeda et al. 1993). Briefly, the unidirectional cDNA library was excised in vivo from the $\lambda$ phage into phagemid DNA using the ExAssist helper phage (Stratagene). Phagemid particles were transfected into E. coli SOLR (Stratagene) and plated on LB-Amp plates to generate plasmid forms. The colonies were randomly selected from the plates and plasmid DNAs were extracted using the Biomek 2000 miniprep system (Beckman, Fullerton, CA, USA). The inserts of the cDNA clones were sequenced from both ends. DNA sequencing was performed using an ABI PRISM BigDye Terminator Cycle Sequencing FS Ready Reaction Kit (Applied Biosystems, Foster City, CA, USA). The sequencing reaction products were analyzed by an Applied Biosystems DNA sequencer model 3700. Quality assessment and base trimming of each sequence 
were performed using PE Sequencing Analysis 3.3 software (Applied Biosystems). Contaminated vector sequences were removed using Assembly LIGN (copyright by Oxford Molecular Group plc). Sequences containing less than $1 \%$ ambiguous bases longer than $200 \mathrm{bp}$ were counted as good sequences.

\section{Database analysis of human pancreatic islet ESTs}

We analyzed $\sim 22000$ 3'-ESTs from human pancreatic islet tumors together with nonredundant nucleotide and peptide sequences extracted in silico from GenBank databases at the National Center for Biotechnology Information (NCBI) (http://www.ncbi.nlm.nih.gov/). We first removed tracks of ambiguous residues from the obtained ESTs and masked the highly repetitive sequences by RepeatMasker (http://ftp.genome. washington.edu/RM/RepeatMasker.html). The resultant sequences were subjected to a BLAST search against a merged database containing daily updates of human sequences from GenBank. The program BLASTN (Altschul et al. 1997) was used to compare the sequences at the level of the nucleic acids. If a query EST sequence shared more than $95 \%$ sequence identity without masked and ambiguous nucleotides and showed a score of more than 235 with any other sequences in the database, it was grouped with the query. If there was at least one sequence in common, groups were merged into a single cluster. An EST sequence that did not belong to any of the clusters is a singleton. To assemble the sequences that belonged in each cluster, we applied the CAP2 program to make contigs (Huang 1996). The EST clones without any match to known genes in the nucleotide database were re-sequenced from the $5^{\prime}$-end. Because the sequences from the $5^{\prime}$-end are more likely to provide protein coding information, the BLASTX program (Altschul et al. 1997) was used to conceptually translate the 5 '-EST sequence in all six reading frames and compare the sequences with those in the peptide database at NCBI. ESTs identical or highly similar to known genes were classified into seven major categories according to the general functions of the proteins encoded, and then further classified into subcategories according to their specific functions. Computer-based
Table 1 Summary of human pancreatic islet tumor 3'-ESTs

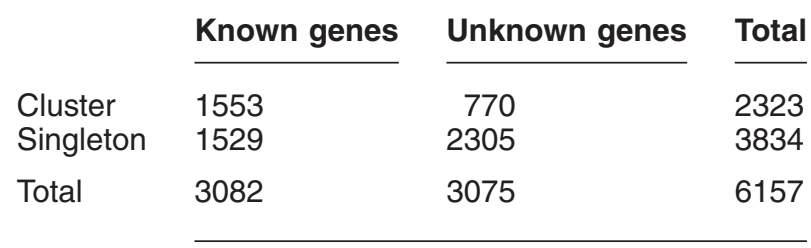

chromosomal localization of the ESTs was also performed using the UniGene database at NCBI, a repository of clustered EST sequences from various tissues and their assigned chromosomes.

\section{Results and discussion}

\section{Characterization of human pancreatic islet tumor ESTs}

A total of $\sim 22000$ random clones from a unidirectional cDNA library were partially sequenced from the $3^{\prime}$-end to generate 21267 sequences with good quality. Such large-scale sequencing generally provides highly redundant ESTs that should be aligned and assembled for a set of unique genes. After 1520 repetitive sequences $(7 \cdot 1 \%)$ and 4764 mitochondrial DNAs (22.4\%) were removed, the remaining 14983 3'-ESTs (DDBJ accession BP383879-398856, BP433025433029) were assembled into non-redundant sequence groups. The clustering analysis generated 6157 non-redundant sequences comprising 2323 groups of sequences and 3834 singletons (Table 1). Of these, 3082 were known genes. Since no genes for major secretory proteins of the exocrine pancreas, such as $\alpha$-amylase, pancreatic lipase, elastase, and trypsinogen, were identified in this study, nearly $100 \%$ of the ESTs obtained should originate from the endocrine pancreas. Relative frequencies of the ESTs for each gene reflect the average level of expression of the corresponding mRNAs in the pooled tissues. Since groups with a redundancy of 1-5 times accounted for $93 \cdot 8 \%$ of the groups, our massive sequencing was clearly effective in identifying a larger number of non-redundant mRNAs expressed at moderate levels (Table 2). Approximately $6 \%$ of the ESTs were identified 6-20 times. Fifty-nine abundant sequences identified more than 20 times $(0 \cdot 8 \%)$ are shown in Table 3 . Of these, guanine nucleotide 
Table 2 Redundancy of nucleotide sequences from the 3 -end of the cDNA clones

\begin{tabular}{lrr}
\cline { 2 - 3 } Redundancy & & \\
1 & 3834 & $62 \cdot 4$ \\
2 & 1123 & $18 \cdot 2$ \\
3 & 458 & $7 \cdot 4$ \\
4 & 226 & $3 \cdot 6$ \\
5 & 136 & $2 \cdot 2$ \\
$6-10$ & 232 & $3 \cdot 7$ \\
$11-20$ & 91 & $1 \cdot 5$ \\
$21-50$ & 42 & $0 \cdot 7$ \\
$51-100$ & 8 & $0 \cdot 1$ \\
$>100$ & 7 & $0 \cdot 1$ \\
& &
\end{tabular}

binding protein $(\mathrm{Gs} \alpha)$ (255 times) and translation elongation factor- $1 \alpha$ (156 times) are most abundant in this library, and were also frequently found in our previous study of normal islets (Takeda et al. 1993). Genes for myosin binding protein C, ferritin heavy and light chains, ribosomal proteins, actin isoforms, and glyceraldehyde-3-phosphate dehydrogenase are also abundant both in normal islets and tumors, suggesting that these genes are essential for cell life. While the highly expressed clones are mostly house-keeping genes, the pancreatic polypeptide (PP) (154 times) and islet amyloid polypeptide (IAPP)/amylin (41 times) genes are specific to $\mathrm{PP}$ and $\beta$ cells respectively. As none of the other genes for islet-specific hormones have been identified at high frequency (glucagon only once, and insulin, somatostatin, and other related hormones have not been found), the tissues used for a cDNA library (Stratagene) should not be functioning hormone-secreting tumors, but IA-1 protein, which is associated with human insulinoma (Goto et al. 1992), is moderately expressed (10 times). Elevated plasma PP levels have been reported in $\sim 50 \%$ of patients with pancreatic islet tumors (Polak et al. 1976), which is consistent with the frequency of $\mathrm{PP}$ found in this study. Transthyretin (25 times) is also specific to liver and pancreatic islets, but its functional properties in islet cells are unknown (Itoh et al. 1992). Sulfonylurea receptor (SUR1), a component of the $\mathrm{K}_{\mathrm{ATP}}$ channel essential for insulin secretion, is also moderately expressed (15 times). High expression of neuroendocrine secretory protein 55 (153 times), prohormone processing enzymes of carboxypeptidase E (82 times), PG2 (37 times), and chromogranin A (38 times) suggest the hormonesecretion machinery of endocrine pancreas. High expression of zinc finger protein (GOS24), c-fos oncogene, basic transcription factor 3, leukemia (CLL)-associated antigen KW-3, and tumor protein TPT1 may be associated with tumorigenesis of the islet cells.

\section{Expression profile of known genes in pancreatic islet system}

ESTs showing identity or high similarity to known genes were classified into seven major categories on the basis of putative general functions of the protein encoded, as described previously. The categories are cell division, cell signaling/communication, cell structure/motility, cell/organism defence, gene/ protein expression, metabolism, and unclassified (Adams et al. 1995, Hwang et al. 1997). In total, 3104 known genes, 21 of which were identified following a BLASTX search as described below, are represented in the classified data set (supplement at http://imcr.sb.gunma-u.ac.jp/lab/ genetics/isletDB.xls.zip). In accord with studies of other tissues, the largest category of genes was gene/protein expression (723 genes, 23.3\%) (Fig. 1). Successively, smaller categories were cell signaling and communication (18.8\%), metabolism $(16 \cdot 1 \%)$, cell structure/motility $(8 \cdot 1 \%)$ cell/ organism defence $(6 \cdot 1 \%)$, and cell division $(5 \cdot 0 \%)$. ESTs lacking sufficient information to be classified constituted the remainder $(22 \cdot 8 \%)$. To further analyze the molecular complexity, each major category was subdivided according to the putative specific functions of the proteins. For example, the largest category, gene/protein expression, was subdivided into eight subgroups (Fig. 2). Of these, transcription factors includes the largest number of non-redundant genes (256 genes), and ESTs for ribosomal proteins were identified most frequently (864 ESTs for 99 different proteins) (see web-site supplement). The transcription factors include ISL1, Beta2/NeuroD1, G/EBP, Pax6, NFкB, HIxB9, etc, which are essential in islet cell differentiation (Habener \& Stoffers 1998, Edlund 2002). As these frequencies are markedly higher than those found in the previous study of normal islets (Takeda et al. 1993), the constellation of these genes suggests association with continuous cell growth. 
Table 3 List of highly redundant CDNA clones

Gene products

\begin{tabular}{|c|c|}
\hline \multirow{2}{*}{\multicolumn{2}{|c|}{ Redundancy }} \\
\hline Gs $\alpha$ & \\
\hline 156 & translation elongation factor 1 alpha \\
\hline 154 & pancreatic polypeptide \\
\hline 153 & neuroendocrine secretory protein 55 \\
\hline 120 & hemoglobin, alpha \\
\hline 113 & myosin binding protein $\mathrm{C}$ \\
\hline 103 & ribosomal protein L3 \\
\hline 89 & actin, gamma 1 \\
\hline 87 & la-associated invariant gamma-chain \\
\hline 82 & carboxypeptidase $\mathrm{E}$ \\
\hline 78 & major histocompatibility complex, class I, B \\
\hline 73 & hemoglobin, alpha 2 \\
\hline 72 & ribosomal protein $\mathrm{PO}$ \\
\hline 65 & ferritin, light polypeptide \\
\hline 62 & zinc finger transcriptional regulator (GOS24) \\
\hline 54 & ribosomal protein $\mathrm{L} 13 \mathrm{a}$ \\
\hline 47 & hemoglobin, alpha 1 \\
\hline 47 & immunoglobulin kappa light chain \\
\hline 46 & ferritin, heavy polypeptide \\
\hline 44 & actin, beta \\
\hline 44 & CD74 antigen \\
\hline 41 & islet amyloid polypeptide \\
\hline 39 & immunoglobulin lambda light chain \\
\hline 38 & chromogranin A \\
\hline 38 & ubiquitin $\mathrm{C}$ \\
\hline 37 & hypothetical $101.4 \mathrm{kDa}$ protein \\
\hline 37 & prohormone converting enzyme (PC2) \\
\hline 35 & glutathione peroxidase 3 \\
\hline 35 & glyceraldehyde-3-phosphate dehydrogenase \\
\hline 34 & 22 kDa actin-binding protein (SM22) \\
\hline 34 & major histocompatibility complex, class I, C \\
\hline 34 & prosaposin \\
\hline 32 & G protein, beta polypeptide 2 -like 1 \\
\hline 32 & ornithine decarboxylase antizyme 1 \\
\hline 30 & actin, alpha 2 \\
\hline 30 & ribosomal protein L6 \\
\hline 30 & testis enhanced gene transcript (BAX inhibitor 1$)$ \\
\hline 29 & interferon, gamma-inducible protein 30 \\
\hline 29 & ribosomal protein L10 \\
\hline 27 & chemokine (C-C motif) ligand 18 \\
\hline 26 & $c-f o s$ \\
\hline 25 & basic transcription factor 3 \\
\hline 25 & thymosin, beta 4 \\
\hline 25 & transthyretin \\
\hline 25 & tumor protein, translationally-controlled 1 (TPT1) \\
\hline 24 & amyloid beta (A4) precursor-like protein 1 \\
\hline 24 & CLL-associated antigen KW-3 \\
\hline 24 & heat shock $70 \mathrm{kDa}$ protein 8 \\
\hline 24 & transmembrane 4 superfamily member 4 \\
\hline 24 & immunoglobulin heavy chain \\
\hline 23 & ribosomal protein L15 \\
\hline 22 & ADP-ribosylation factor 1 \\
\hline 22 & translation initiation factor, SUI 1 \\
\hline 21 & calmodulin-I \\
\hline 21 & dual specificity phosphatase 1 \\
\hline 21 & osteopontin \\
\hline 21 & regulator of G-protein signalling 5 \\
\hline 21 & ribosomal protein $\mathrm{S} 11$ \\
\hline 21 & ribosomal protein S19 \\
\hline
\end{tabular}

\section{Cellular function}

Intracellular transducers

Translation factors

Hormones/growth factors

Intracellular transducers

Carrier proteins/membrane transport

Contractile proteins

Protein synthesis

Cytoskeleton

Immunology

Protein turnover

Immunology

Carrier proteins/membrane transport

Protein synthesis

Carrier proteins/membrane transport

Transcription factors

Protein synthesis

Carrier proteins/membrane transport Immunology

Carrier proteins/membrane transport

Cytoskeleton

Unclassified

Protein turnover

Immunology

Vesicular transport

Posttranslation modification

Unknown

Protein turnover

Homeostasis

Sugar/glycolysis

Cytoskeleton

Immunology

Unclassified

Protein modification

Metabolism

Cytoskeletal

Protein synthesis

Apoptosis

Unclassified

Protein synthesis

Hormones/growth factors

Transcription factors

Transcription factors

Hormones/growth factors

Transport

Unclassified

Protein turnover

Transcription factors

Stress response

Unclassified

Immunology

Protein synthesis

Effectors/modulators

Translation factors

Effectors/modulators

Protein modification

Extracellular matrix

Extracellular matrix

Protein synthesis

Protein synthesis 


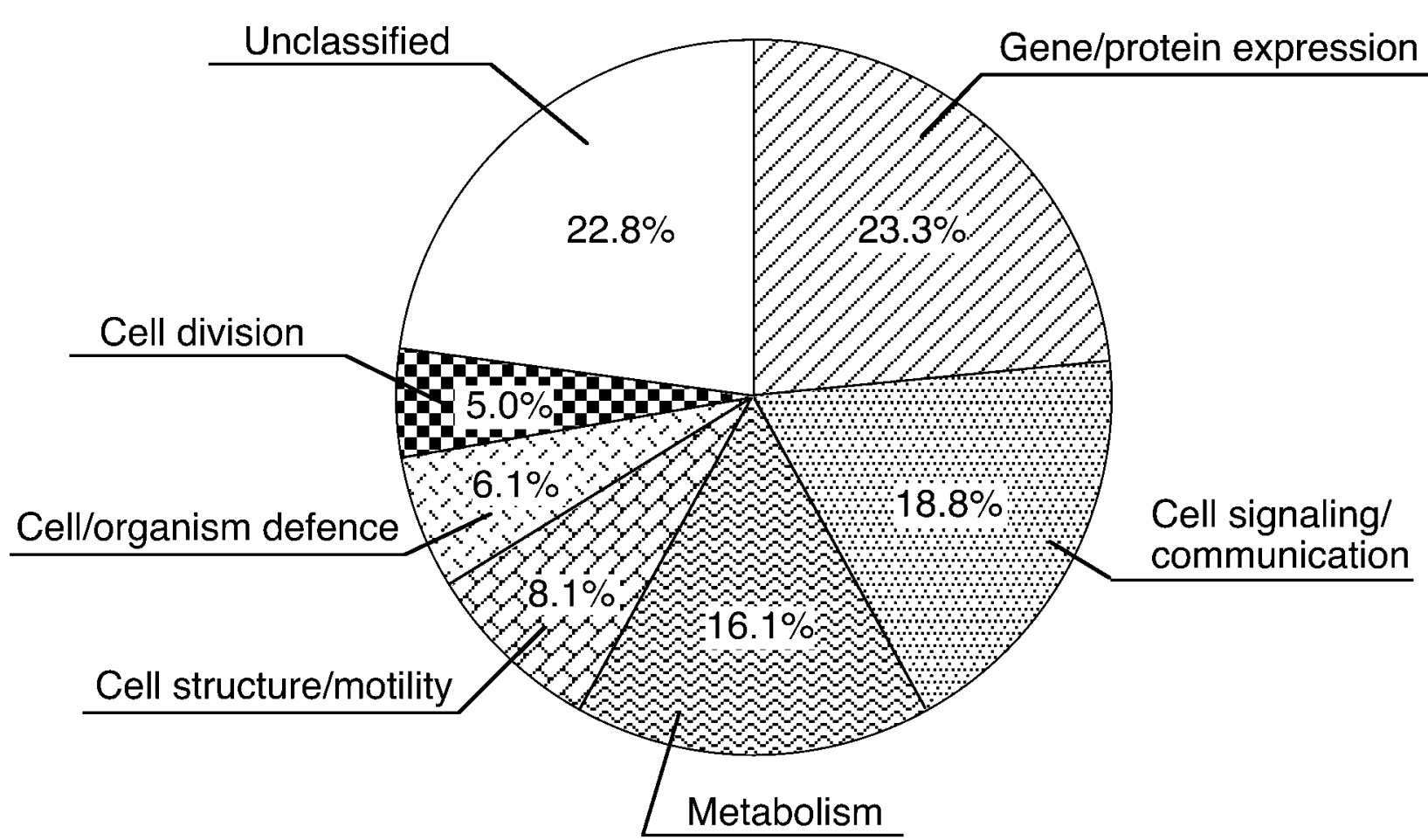

Figure 1 Functional distribution of known genes in pancreatic islet tumors. ESTs showing identity or high similarity to known genes were classified into seven major categories on the basis of the putative general functions of the protein encoded.

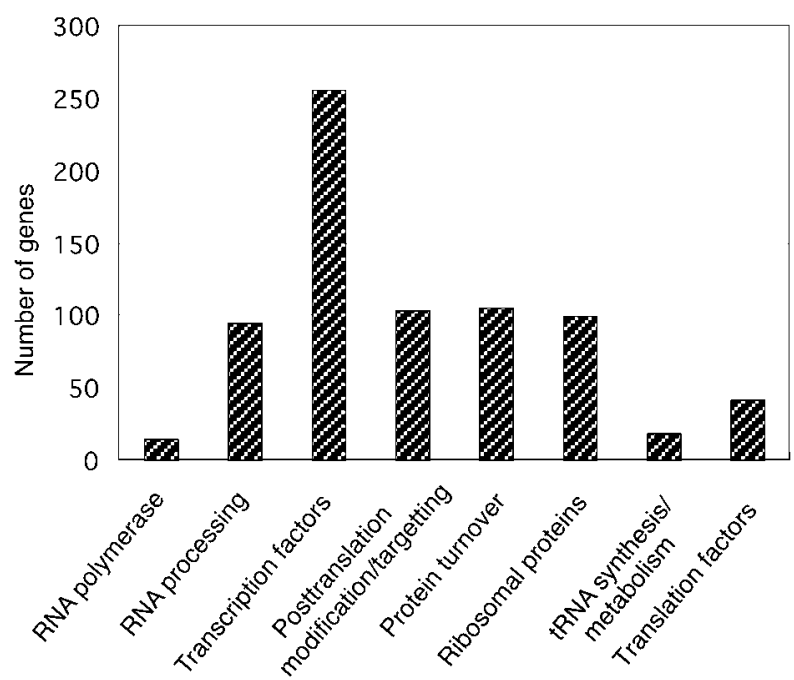

Figure 2 Subclasses of the gene/protein expression category. The largest category in Fig. 1 was subdivided into eight subgroups. Of these, the transcription factors subgroup contains the largest number of non-redundant genes.

\section{Human homologs of known genes and new members of gene families}

In this study, $49 \cdot 9 \%$ of the non-redundant 3 '-ESTs did not match any of the known genes in the nucleotide database. To identify novel human genes encoding proteins structurally related to the known proteins, we performed a BLASTX search in the peptide databases using 4486 5'-ESTs, with a $P$ value of $10^{-7}$ and a similarity of $50 \%$ as the cut off for significant similarity. Thirty-three amino acid sequences predicted from 46 ESTs showed 100\% identity to the known human sequences, indicating that the $3^{\prime}$-end sequences of these clones are not contained in the cDNA sequences deposited in the nucleotide databases. These ESTs, including the 21 new genes, were then assigned to the known groups. Of those remaining, 58 represent human homologs of genes identified in other species or new members of structurally related families (Table 4). Of these, the proteins similar to zinc finger proteins, retrovirus-related POL polyproteins, and modifying enzymes were most abundant. Functional analyses of the proteins identified through this approach 
Table 4 Human homologs of know genes and new members of gene families

\section{Gene name}

\section{Clone}

hbt11524

hbt06299

htp-42-33

h-t-233-25

h-t-224-90

hbt07842

h-t-246-83

hbt10512

h-t-073-11

hbt12379

htp-20-85

htp-54-36

hbt09323

h-t-205-38

htp-22-41

htp-33-34

hbt02542

hbt05127

hbt03746

htp-33-70

htp-02-65

hbt03070

htp-39-25

hbt02830

h-t-220-23

h-t-044-31

htp-30-16

hbt06343

hbt07110

h-t-210-82

h-t-226-50

h-t-216-19

htp-65-42

htp-10-13

h-t-250-63

htp- $41-79$

h-t-220-25

h-t-226-83

hbt00363

htp-28-64

h-t-244-14

h-t-230-61

hbt00553

htp-35-40

htp-15-87

htp-48-41

h-t-268-44

hbt03505

htp-56-60

htp-19-90

hbt10883

h-t-221-06

htp-19-77

hbt07584

htp-49-11

hbt02516

h-t-226-86

hbt01372

mlo2 protein

protein TipD

putative p150 asparagine tRNA ligase

aspartate beta hydroxylase (ASPH)

AU RNA binding protein/enoyl-coenzyme $A$ hydratase

beta-glucuronidase precursor (Beta-G1)

carboxypeptidase X 2 (M14 family)

carotenoid regulatory protein

cysteine-rich protease inhibitor

dihydroxyacetone kinase

disks large-associated protein 4

E3 ubiquitin ligase

ELKL motif kinase

endogenous retrovirus $\mathrm{H}$ element-like protein

endonuclease/reverse transcriptase

ENV polyprotein

erythrocyte membrane-associated giant protein antigen 332

ethanol induced 6

ethanolamine kinase-like protein EK12

glycosyltransferase ALG2

KIAA1076 protein

KIAA1294 protein

KIAA1402 protein

kidney ankyrin repeat-containing protein

LRPR1 homolog 1

muscle protein 534

myeloid-associated differentiation marker (SB135)

neuronal protein

nischarin; imidazoline receptor candidate

pecanex-like protein 1

probable acyl-CoA dehydrogenase

probable membrane protein YGL231c

protein tyrosine phosphatase

putative protein of ancient origin (39.6 kDa)

putative RNA binding protein 1

RAS-like, estrogen-regulated, growth-inhibitor

replication licensing factor MCM2/3/5-type protein

retrovirus-related $\mathrm{POL}$ polyprotein

retrovirus-related POL polyprotein

retrovirus-related POL polyprotein

reverse transcriptase homolog

splicing factor $\mathrm{CC} 1.3$

RRM-containing protein

seizure 6-like protein

$\mathrm{SH} 3$ domain-containing $70 \mathrm{kDa}$ protein

sodium/glucose cotransporter 1

$\mathrm{TBC}, \mathrm{SH}$, and RUN-domain containing protein

TPR domain containing protein family member

TU12B1-TY protein

tubulin tyrosine ligase-like protein HOTTL

WD-repeat protein 18

zinc finger protein 14 (KOX 6)

zinc finger protein 84

zinc finger protein 91

zinc finger protein 135

zinc finger protein 238

zinc finger protein 268
Species

Arabidopsis thaliana

Homo sapiens

Homo sapiens

Homo sapiens

Mus musculus

Mucor circinelloides

Mus musculus

Mesorhizobium loti

Rattus norvegicus

Mus musculus

Homo sapiens

Homo sapiens

Mus musculus

Homo sapiens

Plasmodium yoelii yoelii

Mus musculus

Mus musculus

Dictyostelium discoideum

Homo sapiens

Homo sapiens

Homo sapiens

Homo sapiens

Homo sapiens

Schizosaccharomyces pombe

Mus musculus

Homo sapiens

Felis catus

Homo sapiens

Homo sapiens

Pseudomonas

Saccharomyces cerevisiase

Dictyostelium discoideum

Thermococcus kodakaraensis

Homo sapiens

Caenorhabditis elegans

Rattus norvegicus

Homo sapiens

Caenorhabditis elegans

Mus musculus

Mus musculus

Mus musculus

Homo sapiens

Homo sapiens

Arabidopsis thaliana

Homo sapiens

Homo sapiens

Mus musculus

Caenorhabditis elegans

Caenorhabditis elegans

Homo sapiens

Mus musculus

Homo sapiens

Homo sapiens

Homo sapiens

Homo sapiens

Homo sapiens

Homo sapiens

Homo sapiens

\begin{tabular}{|c|c|}
\hline$\%$ SIM & $P$ value \\
\hline 86 & $2 \mathrm{E}-13$ \\
\hline 77 & $8 \mathrm{E}-43$ \\
\hline 81 & $2 \mathrm{E}-31$ \\
\hline 60 & $1 \mathrm{E}-16$ \\
\hline 61 & $4 \mathrm{E}-28$ \\
\hline 68 & $1 \mathrm{E}-16$ \\
\hline 74 & $1 \mathrm{E}-75$ \\
\hline 58 & $3 E-15$ \\
\hline 75 & $8 E-48$ \\
\hline 95 & $4 \mathrm{E}-74$ \\
\hline 51 & $2 \mathrm{E}-12$ \\
\hline 64 & $2 \mathrm{E}-37$ \\
\hline 61 & $7 \mathrm{E}-14$ \\
\hline 62 & $2 \mathrm{E}-51$ \\
\hline 50 & $6 \mathrm{E}-19$ \\
\hline 64 & $5 \mathrm{E}-27$ \\
\hline 92 & $6 \mathrm{E}-49$ \\
\hline 63 & $8 \mathrm{E}-34$ \\
\hline 60 & $1 \mathrm{E}-40$ \\
\hline 88 & $2 \mathrm{E}-26$ \\
\hline 77 & $2 \mathrm{E}-33$ \\
\hline 72 & $2 \mathrm{E}-21$ \\
\hline 61 & $3 E-18$ \\
\hline 58 & $3 E-07$ \\
\hline 96 & $2 \mathrm{E}-32$ \\
\hline 79 & $2 \mathrm{E}-70$ \\
\hline 85 & $1 \mathrm{E}-44$ \\
\hline 92 & $1 \mathrm{E}-77$ \\
\hline 60 & $4 \mathrm{E}-35$ \\
\hline 87 & $3 \mathrm{E}-18$ \\
\hline 54 & $3 \mathrm{E}-11$ \\
\hline 54 & $4 \mathrm{E}-31$ \\
\hline 63 & $1 \mathrm{E}-11$ \\
\hline 58 & 1E-09 \\
\hline 67 & $9 \mathrm{E}-41$ \\
\hline 58 & $3 \mathrm{E}-12$ \\
\hline 52 & $2 \mathrm{E}-11$ \\
\hline 54 & $7 \mathrm{E}-18$ \\
\hline 62 & $3 \mathrm{E}-14$ \\
\hline 61 & $4 \mathrm{E}-15$ \\
\hline 74 & $9 \mathrm{E}-16$ \\
\hline 54 & $1 \mathrm{E}-10$ \\
\hline 85 & $9 \mathrm{E}-51$ \\
\hline 86 & $3 E-35$ \\
\hline 65 & $2 \mathrm{E}-25$ \\
\hline 65 & $5 \mathrm{E}-10$ \\
\hline 82 & $9 \mathrm{E}-31$ \\
\hline 70 & $6 \mathrm{E}-48$ \\
\hline 72 & $6 \mathrm{E}-33$ \\
\hline 84 & 7E-85 \\
\hline 67 & 3E-75 \\
\hline 87 & $1 \mathrm{E}-113$ \\
\hline 62 & $1 \mathrm{E}-24$ \\
\hline 55 & $4 \mathrm{E}-10$ \\
\hline 57 & $2 \mathrm{E}-54$ \\
\hline 80 & $5 \mathrm{E}-76$ \\
\hline 50 & $6 \mathrm{E}-16$ \\
\hline 91 & $7 \mathrm{E}-51$ \\
\hline
\end{tabular}




\section{Known ESTs: 3082}

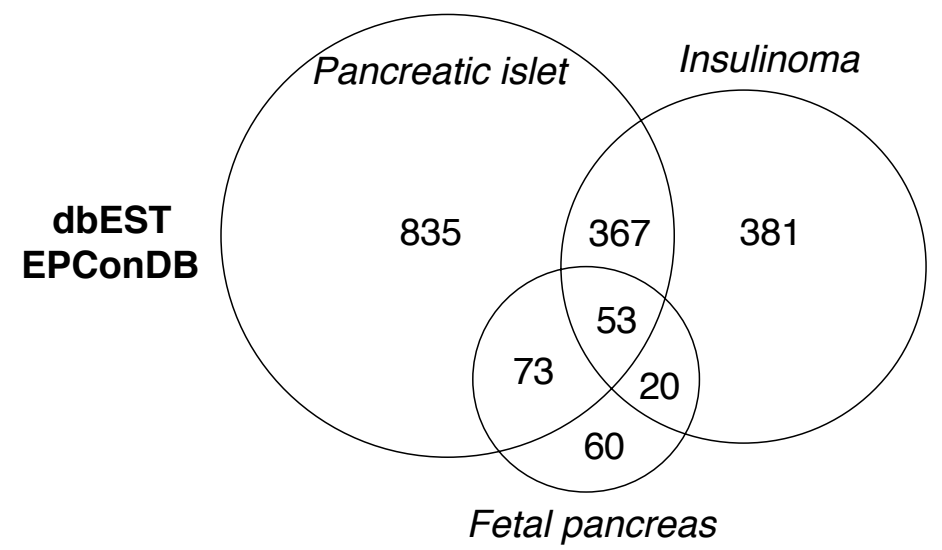

\section{Unknown ESTs: 3075}

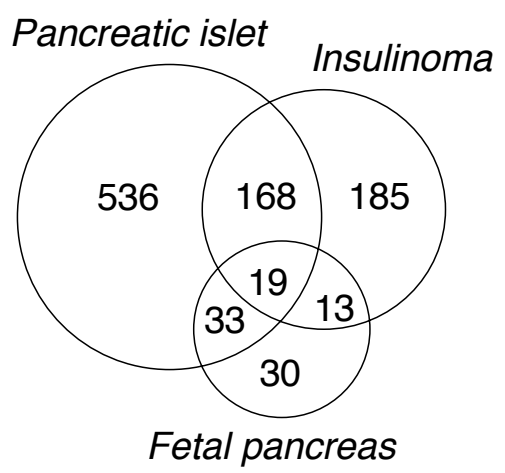

Figure 3 Comparison of non-redundant human islet-cell tumor ESTs with islet-related sequences in dbEST and EPConDB. In total, 36 888, 10 201, and 1368 sequences from human pancreatic islets, insulinomas, and fetal pancreas respectively, are deposited in the databases (Bernal-Mizrachi et al. 2003, dbEST release 041103). In this study, 3082 known genes and 3075 unknown genes were examined. The numbers of sequences with a significant match between the tissues are shown. Of the known and unknown ESTs of this study, 1293 and 2091 respectively show no significant match with any of the islet-related sequences in the databases.

should clarify the role of new members of structurally related families in pancreatic islets. The remaining ESTs (2984 genes) were not related to any other sequences in the databases. As found in similar large-scale cDNA sequencing studies carried out in other tissues, about $50 \%$ of the clones appear to be derived from genes that have not previously been described.

\section{Collection of non-redundant ESTs newly found in endocrine pancreas}

Since a number of the mRNAs might be up- or down-regulated in disease states including diabetes mellitus and islet cell tumors, even in normal physiological conditions, it is especially important to collect as many non-redundant cDNA clones from multiple tissue sources as possible to cover the wider spectrum of expressed genes involved in its pathogenesis. The Endocrine Pancreas Consortium (EPCon) has created four, one, and two human cDNA libraries from normal pancreatic islets, insulinoma, and fetal pancreas respectively, along with the ESTs determined from each library (Bernal-Mizrachi et al. 2003, Kaestner et al. 2003). In total, $\sim 48000$ ESTs from these tissues representing $\sim 9000$ non-redundant sequences
(UniGene) are available in the public EST database (dbEST) at NGBI and EPConDB (http:// www.cbil.upem.edu/EPCoDB/). The 6157 nonredundant ESTs obtained in this study were compared with these islet-related ESTs in dbEST and EPConDB using the BLASTN program. Of 3082 and 3075 known and unknown ESTs, 1789 and 984 respectively matched with sequences in the databases (Fig. 3). As the remainder of the 3384 genes $(55 \%)$ are newly added to the molecular catalog, this characterization of islet-cell tumor cDNAs represents a significant increase in the number of non-redundant ESTs. Altogether, $\sim 13000$ genes of various levels of expression have been found in normal and tumor tissues of human pancreatic islets and fetal pancreas. The newly found genes in this study were further compared with 5041573 EST entries from other human tissues (dbEST release 041103), resulting in the identification of 587 genes with no match to any of the sequences in dbEST. These genes could be specific to pancreatic islets or their expression levels might be very low in other tissues. Large-scale in situ hybridization analysis using these sequences is in progress in our laboratory to determine their tissue distribution. Future analyses of the function of these genes should lead to a better 
understanding of the tissue-specificity and tumorigenesis of endocrine pancreas.

\section{Future directions of genetic studies of diabetes mellitus}

Various mutations in the genes are involved in the more than 3000 genetic disorders so far described in man, and efforts are now being made to map the genes of interest on the genome that are actually expressed in a particular tissue or cell to facilitate more rapid isolation of disease genes.

In this study, we describe a collection of 19469 human pancreatic islet-related ESTs (3'-EST 14983 and 5'-EST 4486) representing 6157 different transcripts; 4764 ESTs for mitochondrial sequences are not included in this collection. Establishment of a molecular catalog of expressed genes will facilitate the development of DNA microarray technology. At present, PanChip microarray, which was prepared using 3400 cDNA sequences from the mouse whole pancreas (Scearce et al. 2002), is only available for the analysis of gene expression in murine pancreas. Accordingly, the human islet-specific EST microarray should be important for the analysis of human diseases using limited amounts of the tissues. Such an effort is in progress in our laboratory. The functional analysis of newly discovered genes through this approach might sufficiently clarify the molecular mechanisms underlying the pathogenesis of diabetes and lead to new therapies for the improvement and regeneration of $\beta$-cell functions through manipulation of gene expression or products. Each of the genes described here was assigned to its chromosome by analysis of the UniGene and PubMed literature databases at NCBI (Table 4). Coupled with integrated information on islet-specific functions, our chromosomal mapping should complement genetic linkage studies and facilitate positional candidate cloning for the identification of diabetogenes in genetically defined regions.

\section{Acknowledgements}

We thank S Oike, R Kawakami, Y Yaginuma, I Uda, and $\mathrm{T}$ Takahashi for excellent technical assistance. This study was supported by Grants-inAid for Creative Scientific Research, for Scientific Research A-C, and for Scientific Research on
Priority Areas C from the Japanese Ministry of Science, Education, Sports, Culture and Technology; Comprehensive Research on the Human Genome, and Tissue Engineering and Food Biotechnology from the Japanese Ministry of Health, Labor and Welfare; the Yamanouchi Foundation for Research on Metabolic Disorders; and the Takeda Science Foundation.

\section{References}

Adams MD, Kelley JM, Gocayne JD, Dubnick M, Polymeropoulos MH, Xiao H, Merril CR, Wu A, Olde B, Moreno RF et al. 1991 Complementary DNA sequencing: expressed sequence tags and human genome projects. Science 252 1651-1656.

Adams MD, Kerlavage AR, Fieischmann RD, Fuldner RA, Bult CJ, Lee NH, Kirkness EF, Weinstock KG, Gocayne JD, White O et al. 1995 Initial assessment of human gene diversity and expression patterns based upon 83 million nucleotides of cDNA sequence. Nature 377 (Suppl) 3-174.

Altschul SF, Madden TL, Schaffer AA, Zhang J, Zhang Z, Miller W \& Lipman DJ 1997 Gapped BLAST and PSI-BLAST: a new generation of protein database search programs. Nucleic Acids Research 25 3389-3402.

Bernal-Mizrachi E, Cras-Meneur G, Ohsugi M \& Permutt MA 2003 Gene expression profiling in islet biology and diabetes research. Diabetes Metabolism Research Reviews 19 32-42.

Edlund H 2002 Pancreatic organogenesis - developmental mechanisms and implications for therapy. Nature Genetics Reviewes 3 524-532.

Gordon CW \& Halban PA 2001 Islet cell hormones: production and degradation. In Principles and Practice of Endocrinology and Metabolism, 3rd ed., pp 1296-1303. Ed. KL Becker. Philadelphia: Lippincott Williams \& Wilkins.

Goto Y, De Silva MG, Toscari A, Prabhakar BS, Notkins AL \& Lan MS 1992 A novel human insulinoma-associated cDNA, IA-1, encodes a protein with 'zinc-finger' DNA-binding motifs. Fournal of Biological Chemistry 267 15252-15257.

Habener JF \& Stoffers DA 1998 A newly discovered role of transcription factors involved in pancreas development and the pathogenesis of diabetes mellitus. Proceedings of the Association of American Physicians 110 12-21.

Huang X 1996 An improved sequence assembly program. Genomics $3321-31$

Hwang DM, Dempsey AA, Wang RX, Rezvani M, Barrans JD, Dai KS, Wang HY, Ma H, Cukerman E, Liu YQ et al. 1997 A genome-based resource for molecular cardiovascular medicine. Toward a compendium of cardiovascular genes. Circulation 96 4146- 4203.

International Human Genome Sequencing Consortium 2001 Initial sequencing and analysis of the human genome. Nature $\mathbf{4 0 9}$ 860-921.

Itoh N, Hanafusa T, Miyagawa J, Tamura S, Inada M, Kawata S, Kono N \& Tarui S 1992 Transthyretin (prealbumin) in the pancreas and sera of newly diagnosed type I (insulin-dependent) diabetic patients. Fournal of Clinical Endocrinology and Metabolism 74 $1372-1377$.

Kaestner KH, Lee CS, Scearce LM, Brestelli JE, Arsenlis A, Le PP, Lantz KA, Crabtree J, Pizarro A, Mazzarelli J et al. 2003 Transcriptional program of the endocrine pancreas in mice and humans. Diabetes 52 1604-1610.

Polak JM, Bloom SR, Adrian TE, Heiz P, Bryant MG \& Pearse AGE 1976 Pancreatic polypeptide in insulinoma, gastrinoma, VIPoma and glucagonoma. Lancet 1 328-330. 
Scearce LM, Brestelli JE, McWeeney SK, Lee CS, Mazzarelli J, Pinney DF, Pizarro A, Stoeckert CJ Jr, Clifton SW, Permutt MA et al. 2002 Functional genomics of the endocrine pancreas. The pancreas clone set and PanChip, new resources for diabetes research. Diabetes 51 1997-2004.

Takeda J, Yano H, Eng S \& Bell GI 1993 A molecular inventory of human pancreatic islets: sequence analysis of 1000 cDNA clones. Human Molecular Genetics 2 1793-1798.
Takeda J, Espinosa R III, Eng S, LeBeau MM \& Bell GI 1995 Chromosomal assignment and tissue distribution of novel expressed sequence tags from a human pancreatic islet cDNA library. Genomics 29 276-281.

Received 27 May 2003

Accepted 13 August 2003 